\title{
Early results for equivalent wavefield transform for active infrared breast thermography
}

\author{
by J. Gershenson*. M. Gershenson ** \\ *Department of Nuclear Medicine, Veterans Heath Association - Grater Los Angeles. California, USA, 90025 \\ **134 Laurel, Cholul, Yucatán, México, 97305
}

\begin{abstract}
Dynamic breast thermogram data is collected by recording the breast IR thermograms sequence following cooling of the breast. Equivalent wave field transform (EWFT) was developed as a tomographic nondestructive evaluation method for testing of materials. We obtained human data from the Brazilian 'Database for Mastology'. The data was originally collected for use in artificial intelligence algorithm and not for time series analysis. Method to extract excitation profile using principal component was developed. Inversion reveals clear depth resolved images. The potential for the method in dense breast is very promising both as classification and as low cost non ionizing screening methods.
\end{abstract}

\section{Introduction}

The purpose of this work is to introduce a new analytical/numerical inversion method which provides threedimensional (3D) sub-surface imaging beneath the skin from time sequenced infrared (IR) thermography images following temperature change. The work was motivated by advances in thermal nondestructive evaluation methods. Breast thermography has been FDA approved as an adjunct method to other screening techniques since 1982. Research methods on the use of thermal imaging to detect breast cancer are quite common; with the number of publications in this area increasing over recent years, along with significant improvements in the IR camera hardware becoming commercially available. Two common breast IR imaging protocols exist, static and dynamic. In static imaging, a single thermogram image is taken at a single time point. In dynamic imaging, a series of thermogram images are taken in a successive time interval, recording a change in the temperature at the surface of the skin after external temperature change. Most work published up to date are based on static thermogram and are based on either the large metabolic rate at the cancerous site which result in very large heat generation or on neovascularization associate with cancer. In spite of the large effort

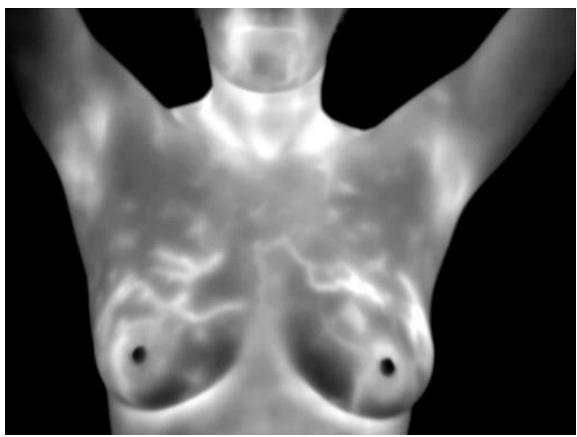

Fig. 1. Static breast thermogram dedicated to static IR imaging the success is moderate. The main reason is the complex interaction between the local heat generation and cooling by the enhance blood flow. The heat outflow is not localized to the cancerous region. Inversion attempts to localize the tumour failed. Dynamic thermogram which is based on change in tissue properties has sufficient information for inversion. Forward modelling confirms the ability to detect cancerous tissue. Inversion of simulated data is reported in the literature, we don't know of any attempt to invert of real data.

\section{Derivation}

\section{Pence bio heat equation}

$$
\nabla k(\boldsymbol{r}) \nabla T(\mathbf{r}, t)-\rho C \cdot \partial / \partial t T(\boldsymbol{r}, t)-\omega C b\left[T(\mathbf{r}, t)-T_{0}(\mathbf{r}, t)\right]=h_{d}(\boldsymbol{r}, t)
$$

\begin{tabular}{|c|c|c|c|c|c|}
\hline & $\begin{array}{c}\text { Density } \\
\mathbf{( k g / m 3 )}\end{array}$ & $\begin{array}{c}\text { Specific heat } \\
(\mathbf{J} / \mathbf{k g ~ K})\end{array}$ & $\begin{array}{c}\text { Thermal conductivity } \\
\mathbf{( W / m )}\end{array}$ & $\begin{array}{c}\mathbf{w b C b} \\
\mathbf{( W / m 3} \circ \mathbf{C})\end{array}$ & $\begin{array}{c}\mathbf{Q m} \mathbf{~ ( H d ) ~} \\
\mathbf{( W / m 3 )}\end{array}$ \\
\hline Subcutaneous fat & 930 & 2770 & 0.21 & 800 & 400 \\
\hline Gland & 1050 & 3770 & 0.48 & 2400 & 700 \\
\hline Tumor & 1050 & 3770 & 0.48 & 48,000 & 65,400 \\
\hline Blood & 1100 & 3300 & 0.45 & & \\
\hline
\end{tabular}

Table 1: Thermophysical parameters of typical breast ${ }^{2}$

The main difference between healthy and disease tissues are the large heat generation Qm (hd) and the large perfusion $\omega \mathrm{Cb}$. 
The wave equation

$$
\nabla^{2} X(\mathbf{r}, t)-v^{-2} \cdot \partial^{2} / \partial t^{2} X(\boldsymbol{r}, t)=h_{w}(\boldsymbol{r}, t)
$$

\section{thermal equivalent.}

Using Schouten Van der Pol theorem, we obtain a relation between the equivalent wave field and the temperature.

$$
\begin{gathered}
T(t)=\int_{0}^{\infty} W(t, \tau) X(\tau) d \tau \\
W(t, \tau)=\mathcal{L}^{-1}\left\{\mathrm{e}^{-g(s) \tau} \mathcal{L} h_{d}(s) / \mathcal{L} h_{w} \sqrt{[s+\omega C b / \rho C]}\right\}
\end{gathered}
$$

Derivation is based on Laplace transform in time of both the bio heat equation and the wave equation, equating terms and performing analytical inverse transform.

In discrete time domain, the above equation is a simple matrix multiplication

\section{$T=W X$}

To obtain $\boldsymbol{X}$ out of $\boldsymbol{T}$ we have to invert $\boldsymbol{W}$. When going from wave to diffusive propagation we lose the high frequency information, the inverse transformation is an ill posed one. Inverting the matrix is done by truncated singular value decomposition or Tikhonov methods.

\section{Bio thermal reflectors}

Blood vessels which are good heat sink represent a large inverting reflector. Reflection of high perfusion diseased tissue in the equivalent wave domain is model as analogue electrical two layers impedances and calculating the reflection due to impedance mismatch and then substituting $p^{2}$ for $p$ to obtain the response at the equivalent wave domain

$$
\mathrm{R}(p)=\frac{\sqrt{p^{2}+\left(\omega_{d}-\omega_{d p}\right) / \rho}-p}{\sqrt{p^{2}+\left(\omega_{d}-\omega_{d p}\right) / \rho}+p}
$$

Reflection from diseased tissue is almost as strong as from blood vessels.

\section{Cooling profile extraction}

To perform the inversion, we have to know the heat profile $h_{d}(\boldsymbol{t})$. The data at hand it was recorded only from the end of the cooling period. We used principal component thermography ${ }^{3}$ (PCT) to obtain the excitation profile. With the available breast data, only short section of the response is available when the data is recorded so the method used in NDE is not practical. Calculation was as follow: First we calculate EWFT using a guessed excitation profile, determine the eigen vectors using PCT and then convolve them with the guessed profile result in improved delayed version of the profile.

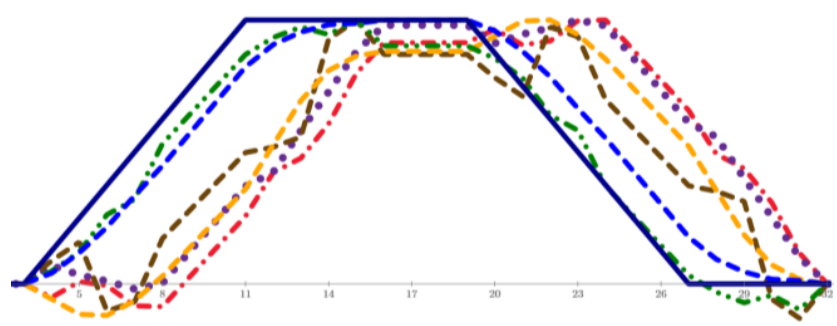

Figure 2: Recovered cooling profiles with the trial one (full line). Profile is slightly longer then the cooling profile reported in the data set 


\section{Mastology data}

We have used data of opportunity from the Brazilian mastology thermal imaging set. The data was taken after blowing air by a fan for a period of about three minutes. Timing of the air flow was not recorded. After motion compensation and cooling profile extraction we inverted the data. Slightly better images were obtained by using principal components analysis following the the inversion which are presented here. Data shown is patient \#282.

We have used data of opportunity from the Brazilian mastology thermal imaging set. After motion compensation and cooling profile extraction we inverted the data. Slightly better images were obtained by following with principal components analysis after the inversion which are presented here
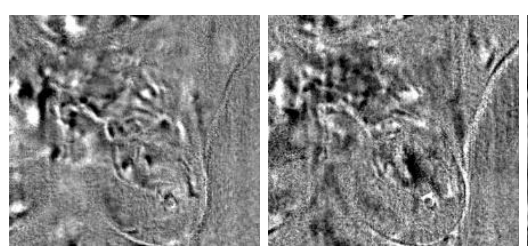
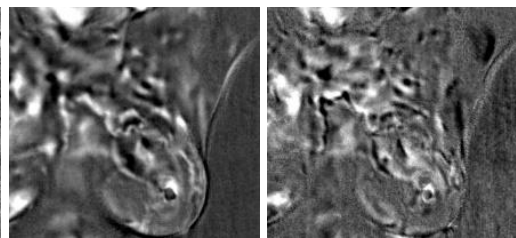

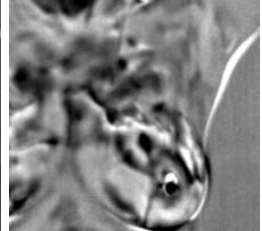

Figure 3: Recovered Eigen-images ordered by depth.

\section{Discussion}

We do not have sufficient information for any medical conclusion out of the above images, but defiantly can recognize blood vessels. We can also identify the same vessels in the static image, but the resolution is better in the dynamic images. The most important conclusion out of this paper is that in spite of the limitations of the data we succeed to obtain consistant data inversion that looks very promising.

\section{Acknowledgment}

Thanks to Visual Lab Brazil for the mastology data.

\section{REFERENCES}

[1] L. F. Silva; D. C. M. Saade; G. O. Sequeiros; A. C. Silva; A. C. Paiva; R. S. Bravo and A. Conci, "A New Database for Breast Research with Infrared Image, Journal of Medical Imaging and Health Informatics", 4 (1), 2014. http://visual.ic.uff.br/en/proeng/

[2] Satish G. Kandlikar, Isaac Perez-Raya, Pruthvik A. Raghupathi, Jose-Luis Gonzalez-Hernandez, Donnette Dabydeen, Lori Medeiros, Pradyumna Phatak "Infrared imaging technology for breast cancer detection Current status, protocols and new directions" International Journal of Heat and Mass Transfer, May 2017

[3] Rajic, N. Principal component thermography for flaw contrast enhancement and flaw depth characterization in composite structures. Composite structures, 58(4), 2002 